\title{
Foreword: New directions in lifelong learning
}

\author{
Paul Morris* - Professor of Comparative Education, \\ UCL Institute of Education, UK
}

In 2007 the Danish Pedagogical University (DPU), Deusto University in Spain and UCL Institute of Education (IOE, now part of University College London) took in the first cohort of students for a Joint Masters Degree in Lifelong Learning and Management. The majority of students were funded by scholarships from the European Union via their Erasmus Scheme for a period of five years. The programme involved students following a two year programme where they began their studies in either DPU or UCL IOE, then they all spent a semester in Deusto and could undertake their dissertations in one of the three universities. Subsequently, the programme was revised to include Melbourne University and was funded for a further five years. The inclusion of Melbourne University allowed students the option of completing one module there.

The multi-site structure of the programme provided the students with experiences in countries with very different cultures and educational approaches. The two-year duration of the Full Time programme and the fact that students were on scholarships gave the students the time and space to develop personally and academically; this was reflected in the quality of their work, especially their dissertations, many of which were published. The selection of students from both across the globe, from large fields of applicants and primarily from those who had working experience was reflected in the overall calibre and multicultural diversity of the student cohorts. There is a great deal written as to the potential benefits of internationalization for students, staff and universities but in practice those benefits often remain primarily aspirational. That was not the case with this programme; the benefits to the staff of working with such a diverse range of mature, bilingual, motivated and diverse international students were palpable; they brought with them an impressive level of energy and intellectual curiosity. In parallel the students greatly benefited from the close and mutually supportive communities that characterized most of the cohorts.

The operation and administration of the programme also required all the institutions involved to learn to operate outside their normal comfort zones and taken for granted modes of operation. The institutions operated with very different: systems of governance; approaches to student selection, progress and assessment; and to pedagogy that required a wide range of unanticipated issues and problems to be regularly addressed. We are greatly indebted to the various programme directors in all of the institutions over the decade, especially in the early years, for working together in an ongoing process of problem-solving to ensure the students' experience was not adversely affected.

In that decade more than 300 students graduated from the programme, and they have gone on to pursue a wide range of careers, including as teachers, researchers, policymakers, writers and entrepreneurs. A significant number of the graduates went on to study for PhDs on topics they began to explore in the Masters programme and they are now working in academia. This volume is made up of articles authored or coauthored by students who studied the programme and brings together a selection of their ongoing scholarship. It reflects the quality of the work undertaken by the students 
in a form that is not captured by the standard evaluation instruments used by both universities and the European Union. The specific focus of this special issue on aspects of lifelong learning is especially appropriate given that it is central to the Sustainable Development Goals promulgated by the United Nations in 2015.

Fittingly, as testament to the calibre of the intake to the programme, this volume was wholly initiated and put together by two students who were members of the last cohort of the programme, one of whom is now undertaking a PhD at UCL IOE. When initially approached I was sceptical as to whether this project would materialize; I am impressed and delighted they have proved me wrong. 